\title{
INFLUÊNCIA DE TRÊS PRÉ-TRATAMENTO DE SUPERFÍCIE COM POSTERIOR APLICAÇÃO DE TINTA POLIÉSTER NA RESISTÊNCIA A CORROSÃO DO AÇO AISI 1008
}

\author{
Patrícia Marcolin ' \\ Leticia Pederiva ${ }^{2}$ \\ Débora Guerra Salvador ${ }^{2}$ \\ Lílian Vanessa Rossa Beltrami ${ }^{3}$ \\ Claúdia Trindade Oliveira ${ }^{4}$ \\ Sandra Raquel Kunst ${ }^{4}$
}

\section{Resumo}

O objetivo deste estudo é analisar a influência de diferentes pré-tratamentos de superfície (fosfato, nanocerâmico e silano) na resistência à corrosão do aço AISI 1008, visando uma posterior aplicação da tinta poliéster. Os pré-tratamentos foram depositados sobre o substrato de maneira distinta. $O$ fosfato teve sua aplicação em escala industrial por meio de pulverização em um sistema onde o desengraxe foi realizado ao mesmo estágio da fosfatização. No nanocerâmico utilizou-se o método por imersão com pH controlado em torno de 4,5 em uma solução de ácido hexafluorzircônio. Para o silano utilizou-se o método de dip-coating, para a aplicação dos precursores tetraetoxisilano (TEOS) e 3-(trimetóxisililpropil) metacrilato (MPTS) pré-hidrolizados. As amostras pré-tratadas foram caracterizadas quanto ao comportamento morfológico e eletroquímico. Após a aplicação de tinta poliéster, as amostras foram avaliadas quanto ao seu comportamento mecânico. Os resultados das análises para os pré-tratamentos superficiais e para os sistemas pré-tratamentos + tinta mostraram que o silano apresentou a melhor resistência à corrosão em relação ao sistema nanocerâmico e fosfatização.

Palavras-chave: Fosfatização; Silano; Nanocerâmicos; Tinta poliéster; Corrosão.

\section{INFLUENCE OF THREE SURFACE PRE-TREATMENTS WITH SUBSEQUENT APPLICATION OF POLYESTER INK IN CORROSION RESISTANCE OF AISI I008 STEEL}

\begin{abstract}
The objective of this study was to analyze the influence of different surface pre-treatments (phosphate, nano-ceramic and silane) on the corrosion resistance of AISI 1008 steel, with a view to the subsequent application of polyester ink. The pre-treatments were deposited on the substrate in distinct ways. Phosphate was applied on an industrial scale by spraying in a system where degreasing was carried out at the same stage of phosphating. In the nano-ceramic pre-treatment the immersion method was used, with controlled $\mathrm{pH}$ of about 4.5 in a solution of hexafluorzirconium acid. For the silane pre-treatment the dip-coating method was used for the application of pre-hydrolysed tetraethoxysilane (TEOS) and 3-(trimethylsilylpropyl) methacrylate (MPTS) precursors. The pre-treated samples were characterized for morphological and electrochemical behavior. After the application of polyester ink, the samples were evaluated for their mechanical behavior. The results for the surface pre-treatments and for the pre-treatment + ink systems showed that silane presented the best corrosion resistance amongst the tested pre-treatments.
\end{abstract}

Keywords: Phosphatization; Silane; Nano-ceramics; Polyester ink; Corrosion.

'Programa de Pós-graduação em Engenharia de Processos e Tecnologias - PGEPROTEC, Universidade de Caxias do Sul - UCS, Caxias do Sul, RS, Brasil.

2Laboratório de Corrosão - LCOR, Universidade de Caxias do Sul - UCS, Caxias do Sul, RS, Brasil.

${ }^{3}$ Laboratório de Pesquisa em Corrosão - LAPEC, Universidade Federal do Rio Grande do Sul - UFRGS, Porto Alegre, RS, Brasil.

${ }^{4}$ Instituto de Ciências Exatas e Tecnológicas - ICET, Universidade Feevale, Novo Hamburgo, RS, Brasil. E-mail: tessaro.sandra@gmail.com

2176-1523 (C) 2017 Associação Brasileira de Metalurgia, Materiais e Mineração. Publicado pela ABM. Este é um artigo de acesso aberto distribuído sob os termos da licença Creative Commons CC BY-NC-ND (Attribution-NonCommercial-NoDerivs) - https:// creativecommons.org/licenses/by-nc-nd/4.0\%. 


\section{INTRODUÇÃO}

Industrialmente, o processo de corrosão de materiais metálicos, como o aço AISI 1008, implica na inativação de equipamentos ou estruturas, promovendo custos para sua substituição ou manutenção [I]. Os principais fatores do meio que influenciam no processo de corrosão de metais são $\circ \mathrm{pH}$ baixo, presença de sais e óxidos dissolvidos (cloretos, sulfetos, entre outros), sólidos em suspensão, elevada temperatura, microrganismos e matéria orgânica [2]. Para retardar este fenômeno e manter o aço estável é necessário modificar a superfície de tal forma que a mesma permaneça isolada do meio externo. Para isso, os processos químicos comumente utilizados na indústria são a cromatização e fosfatização [3] com posterior aplicação de tinta. No entanto, a fosfatização substitui a cromatização apenas quando estamos comparando a aderência da tinta aplicada. Tratamentos superficiais à base de cromatos proporcionam uma excelente proteção contra corrosão e aumento na vida útil da peça. Porém, este processo apresenta efeitos tóxicos e carcinogênicos e envolve altos custos no tratamento de efluentes, o que tem impulsionado as pesquisas com o objetivo de encontrar alternativas que substituam este processo, sendo a fosfatização uma alternativa comumente utilizada $[4,5]$. Porém a fosfatização requer um elevado consumo de água, energia e rigorosos processos de tratamento do efluente gerado. Com isso, investe-se em pesquisas de novos pré-tratamentos que apresentem qualidade igual ou superior à fosfatização e que não ocasione impacto nocivo ao meio ambiente além de promoverem a aderência entre o polímero orgânico (tinta) e a superfície inorgânica, garantindo resistência a corrosão, como por exemplo os pré-tratamentos nanocerâmicos e silanos [6].

Pré-tratamentos nanocerâmicos à base de zircônio se mostraram promissores na proteção contra a corrosão de metais e os pré-tratamentos à base de precursores de silanos, também são uma alternativa promissora, são conhecidos por aumentar a resistência à corrosão do substrato metálico, além de melhorar a adesão de revestimentos orgânicos. Filmes híbridos à base de precursores de silanos, como tetraetoxisilano (TEOS) e 3-(trimetóxisililpropil)metacrilato (MPTS), podem ser facilmente obtidos pelo processo sol-gel, a baixa temperatura, tornando o processo prático e de baixo custo $[7,8]$. Além disso, neste processo pode-se controlar facilmente a estequiometria da reação, possibilitando a formação de filmes de alta pureza e homogeneidade sobre o metal após a completa hidrólise da solução $[9,10]$. Estes pré-tratamentos são desenvolvidos em temperatura ambiente, não contêm metais pesados e são economicamente viáveis $[11,12]$. Nesta perspectiva, o presente estudo tem como objetivo avaliar a influência da resistência à corrosão do aço AISI 1008 quando submetido a três diferentes pré-tratamentos, sendo o fosfato de ferro, utilizado industrialmente e os outros dois, nanocerâmico e silano, como alternativas industriais. Para isso, amostras com diferentes pré-tratamentos foram analisadas em relação as suas propriedades morfológicas, físico-químicas, eletroquímicas e posterior aplicação da tinta poliéster os sistemas foram avaliados quanto ao seu comportamento mecânico. Os resultados dos pré-tratamentos de silano e nanocerâmico foram comparados com as análises do processo por fosfatização e com a amostra sem pré-tratamento.

\section{MATERIAIS E MÉTODOS}

\section{I Limpeza Mecânica e Química do Substrato}

Para aplicação do fosfato de ferro, as amostras de aço AISI 1008 foram encaminhadas a uma linha de pintura industrial que possui o estágio de desengraxe e fosfatização unificado, que consiste na pulverização de $1 \%$ de saloclean $652 \mathrm{~S}$ e $3 \%$ de salofós $753 \mathrm{~S}$, durante 15 minutos, a $50^{\circ} \mathrm{C}$. Para os demais pré-tratamentos, os substratos de aço AISI 1008 foram lixados com lixas d'água de três granulometrias diferentes, sendo elas 320, 360 e 1000, após foram submetidos à limpeza química por meio de desengraxe alcalino realizado em laboratório com imersão em $5 \%$ de saloclean 679 RZ, a $80^{\circ} \mathrm{C}$ por 15 minutos.

\subsection{Aplicação dos Pré-tratamentos}

A aplicação do pré-tratamento à base de fosfato de ferro ocorreu juntamente com o processo de desengraxe em uma linha de pintura industrial. Após, as amostras foram pulverizadas com água corrente e colocadas na estufa de secagem, a $120^{\circ} \mathrm{C}$ por 8 minutos. A aplicação do nanocerâmico foi realizada com a imersão das amostras em uma solução contendo $0,5 \%$ de Nanotex I6R, pH 4,5, à temperatura ambiente $\left(25^{\circ} \mathrm{C}\right)$ por I minuto. Após, as amostras foram retiradas da solução, enxaguadas com água corrente $\mathrm{e}$ secas com ar frio. Para o pré-tratamento à base de silano foram utilizados os precursores tetraetóxisilano (TEOS) e 3-(trimetóxisililpropil) metacrilato (MPTS) com concentração de $24,59 \%$ e $4,17 \%$ respectivamente. Como solvente utilizou-se $16,274 \%$ de água e $54,966 \%$ de álcool. Após a solução foi hidrolisada por 24 horas e obteve-se um pH de 4,25 $( \pm 0,2)$. As amostras foram imersas na solução pelo método de dip-coating, com velocidade controlada de $2,5 \mathrm{~cm} / \mathrm{min}$ durante I minuto. O filme formado permaneceu exposto ao ambiente por I hora e após promoveu-se a cura em estufa na temperatura de $90^{\circ} \mathrm{C}$ por I hora.

Depois da aplicação dos pré-tratamentos ao aço AISI 1008, foi aplicada, por spray, a tinta poliéster, formando o sistema (pré-tratamento + tinta) com a finalidade de verificar em qual deles ocorre a melhor aderência da tinta e consequentemente, melhor desempenho quanto a resistência à corrosão. 


\subsection{Caracterizações dos Pré-tratamentos}

Os pré-tratamentos foram analisados quanto à morfologia, em vista de topo, ao microscópio eletrônico de varredura com uso de microscópio marca Tescan modelo Mira 3. Para as análises eletroquímicas, foi utilizado um potenciostato galvanostato Ivium modelo Ivium Stat. As análises foram realizadas em uma célula de três eletrodos (contra-eletrodo de platina, eletrodo de referência de calomelano saturado e o eletrodo de trabalho, neste caso 0 aço AISI 1008), em eletrólito de $\mathrm{NaCl} 0,05 \mathrm{M}$, sendo a área exposta do eletrodo de trabalho de $0,739 \mathrm{~cm}^{2}$. O potencial de circuito aberto foi monitorado durante os primeiros 500 segundos de imersão e as curvas de polarização foram obtidas com velocidades de varredura de $1 \mathrm{mV} . \mathrm{s}^{-1}$, em uma faixa de potencial de $-200 \mathrm{mV}$ até $450 \mathrm{mV}$ com relação ao potencial de circuito aberto. As medidas de espectroscopia de impedância eletroquímica foram realizadas no potencial de circuito aberto, com aplicação de sinal senoidal de $10 \mathrm{mV}$ em torno do potencial de corrosão e varredura de $100 \mathrm{kHz}$ a $10 \mathrm{mHz}$, conforme outro estudo realizado neste tema, comprovando que está frequência é adequada para verificar os fenômenos de interface $[13,14]$.

A hidrofobicidade dos revestimentos foi avaliada por meio do ângulo de contato pelo método da gota séssil. Neste método, com uso de uma seringa B-D Yale 3D, uma gota é depositada sobre a superfície pré-tratada da amostra e observa-se o ângulo formado pela gota, por meio de fotos, utilizando lente de baixo aumento. Os resulatdos obtidos são uma média dos valores medidos em três pontos diferentes sobre a mesma amostra. As medidas de espectroscopia de infravermelho foram realizadas em um espectrofotômetro Spectrum ASCII I00 para os três revestimentos.

Para a caracterização dos sistemas todos os ensaios foram realizados em triplicata.

\subsection{Caracterizações dos pré-tratamentos com aplicação de tinta poliéster}

As amostras pré-tratadas com aplicação da tinta foram caracterizadas mecanicamente por meio dos ensaios de aderência, impacto e flexibilidade. A resistência ao impacto foi analisada com uso do equipamento BYK-Gardner ISO Impact Tester, onde as amostras foram fixadas no suporte e após foram submetidas a queda de uma massa de $2 \mathrm{~kg}$ lançada de uma altura de $100 \mathrm{~mm}$, para promover uma força brusca e repentina. A resistência à flexibilidade do material foi qualificada utilizando o mandril cônico BYK-Gardner modelo Smg I4I6.

Para a caracterização dos sistemas com aplicação de tinta poliéster todos os ensaios foram realizados em triplicata.

\section{RESULTADOS E DISCUSSÕES}

\section{I Caracterização dos Pré-tratamentos}

A Figura I apresenta as micrografias do aço AISI 1008 lixado, e dos revestimentos estudados. Observa-se que o aço AISI 1008 apresenta rugosidades características do processo de lixamento na superfície da amostra (Figura la).

O pré-tratamento de fosfato de ferro (Figura Ib), apresentou uma superfície menos irregular do que $\circ$ aço lixado e pequenos núcleos de cristais característicos deste tratamento [15]. Apesar dos cristais de fosfato de ferro crescerem tanto em sentido longitudinal como lateral, não se observa a presença de um filme uniforme. Sendo assim, as falhas características da fosfatização fazem com que regiões do metal entrem em contato com o meio, o que comprova a necessidade da aplicação de tinta após a fosfatização para aumentar a resistência do metal aos processos de corrosão, comprovando que a fosfatização tem como finalidade melhorar a aderência de tinta, não interferindo na resistência a corrosão do substrato [16].

O filme formado pelo pré-tratamento à base de nanocerâmico (Figura Ic) mostra cristais e placas com padrões ondulados finos e irregulares, característicos do uso de nanocerâmicas em pré-tratamentos [17]. Além disso, o filme não apresenta micro-fissuras, as quais poderiam influenciar negativamente na resistência à corrosão, criando caminhos preferenciais para permeação do eletrólito através do filme [18]. Outra característica em destaque é o fato da morfologia do filme nanocerâmico apresentar os sulcos formados no processo de polimento, o que sugere que sua
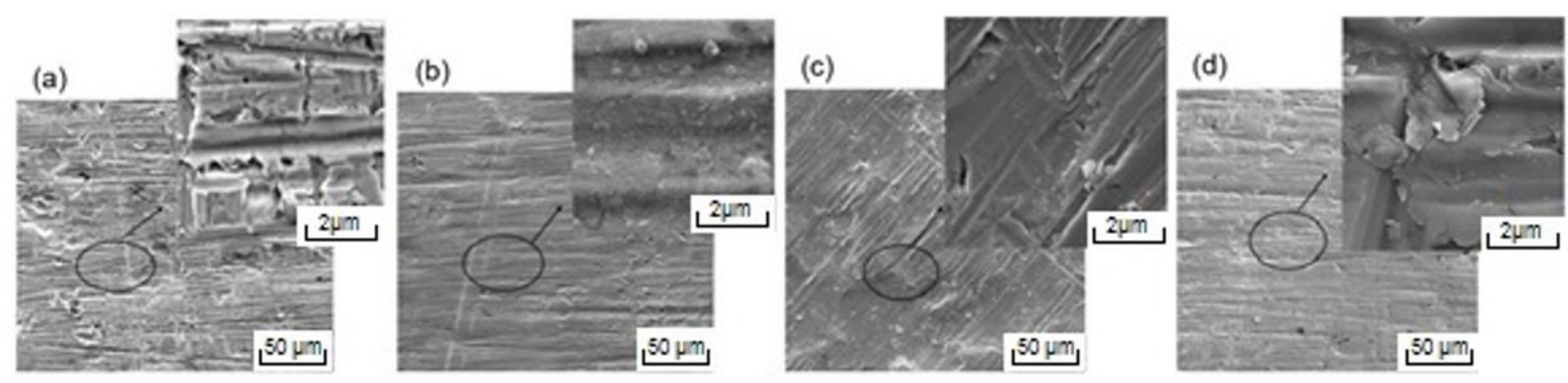

Figura I. Micrografias do aço AISI 1008 (a) lixado (b) com pré-tratamento de fosfato de ferro (c) com pré-tratamento de nanocerâmico e (d) com pré-tratamento de silano. 
expessura é baixa, formada essencialmente pela a coalescência das partículas de óxido de zircônio presentes [18].

Para o pré-tratamento de silano (Figura Id), observa-se a formação de um filme sem fissuras, esta característica homogênea pode estar associada à formação de grupos silanóis na fase gel durante o processo de cura do filme, que foi realizado a $90^{\circ} \mathrm{C}$, superior aos relatados na literatura que indicam que os melhores resultados morfológicos e eletroquímicos foram obtidos a temperatura de cura a $60^{\circ} \mathrm{C}$, sendo assim a cura realizada a $90^{\circ} \mathrm{C}$ caracterizou a adesão efetiva entre o revestimento e o substrato $[9,19]$.

A Figura 2 mostra os resultados da análise do ângulo de contato para os pré-tratamentos. Observa-se que o nanocerâmico e o silano apresentaram maior valor de ângulo de contato em relação ao aço lixado, indicando que os filmes diminuíram a molhabilidade da superfície. $O$ aço lixado e o pré-tratamento de fosfato apresentaram valores próximos, $37^{\circ}$ e $38^{\circ}$ respectivamente. Esse resultado pode estar relacionado com a não uniformidade do filme de fosfato na superfície, conforme discutido na análise da Figura Ib, promovendo o caráter mais hidrofilico, assemelhando-se ao substrato sem pré-tratamento [20]. No entanto o pré-tratamento nanocerâmico, conforme discutido na micrografia por MEV, não apresentou micro-fissuras e a sua morfologia homogênea pode ter influenciado no comportamento mais hidrofilico em relação ao filme de silano, o qual apresentou mais irregularidades (Figura I) [2I].

O pré-tratamento de silano apresentou o maior valor médio para o ângulo de contato em relação aos demais. Os silanos, quando suficientemente reticulados (cross-linking), apresentam caráter hidrofóbico, o que reduz a taxa de permeabilidade da água e íons, protegendo o substrato sobre o qual estão depositados [22]. No entanto, o ângulo de contato de um filme a base de silano bem reticulado é de aproximadamente $90^{\circ}$ e como observado na Figura 2, o valor encontrado foi próximo a $76^{\circ}$. Este valor menor pode estar associado à instabilidade dos grupos $\mathrm{Si}-\mathrm{O}-\mathrm{Si}$, submetidos a reações de hidrólise quando imersos em uma solução aquosa, formando novamente os grupos $\mathrm{Si}-\mathrm{OH}$, hidrofílicos, de tal forma que a água e os íons podem penetrar e reagir com a interface [19].

Nas análises de FTIR, foi avaliada a composição química dos filmes de fosfato, nanocerâmico e silano para verificar a influência dos grupos $\mathrm{OH}$ nas irregularidades observadas nas micrografias obtidas ao MEV. A deformação axial do grupo $\mathrm{OH}$ é caracterizado pela banda situada entre 3200 e $3700 \mathrm{~cm}^{-1}$. Os resultados encontram-se na Figura 3. O espectro do filme de fosfato (Figura 3a), apresenta bandas em 1728 e $1622 \mathrm{~cm}^{-1}$, associadas às vibrações de alongamento dos grupos $\mathrm{C}=\mathrm{O}$ e $\mathrm{C}=\mathrm{C}$, à banda característica de fosfato entre $1000 \mathrm{~cm}^{-1}$ e $1100 \mathrm{~cm}^{-1}$ e à deformação axial referente ao grupo $\mathrm{OH}$ na região entre 3200 e $3700 \mathrm{~cm}^{-1}[9,23]$. Para o espectro do filme nanocerâmico (Figura 3b) os picos em $2900 \mathrm{~cm}^{-1}$ estão associados ao alongamento simétrico e assimétrico das ligações $\mathrm{CH}\left(\mathrm{CH}_{2}\right.$ e $\left.\mathrm{CH}_{3}\right)$ e as demais bandas são similares às encontrados no filme de fosfato citado anteriormente.

Para o filme de silano (Figura 3c), as bandas fortes entre 1000 e $1200 \mathrm{~cm}^{-1}$ estão atribuídas a ligações $\mathrm{Si}-\mathrm{O}-\mathrm{Si}$ sendo estas as principais ligações da estrutura do filme. A ampla absorção entre $3200 \mathrm{~cm}^{-1}$ e $3700 \mathrm{~cm}^{-1}$ é característica da deformação axial do $\mathrm{OH}$, que pode surgir a partir de

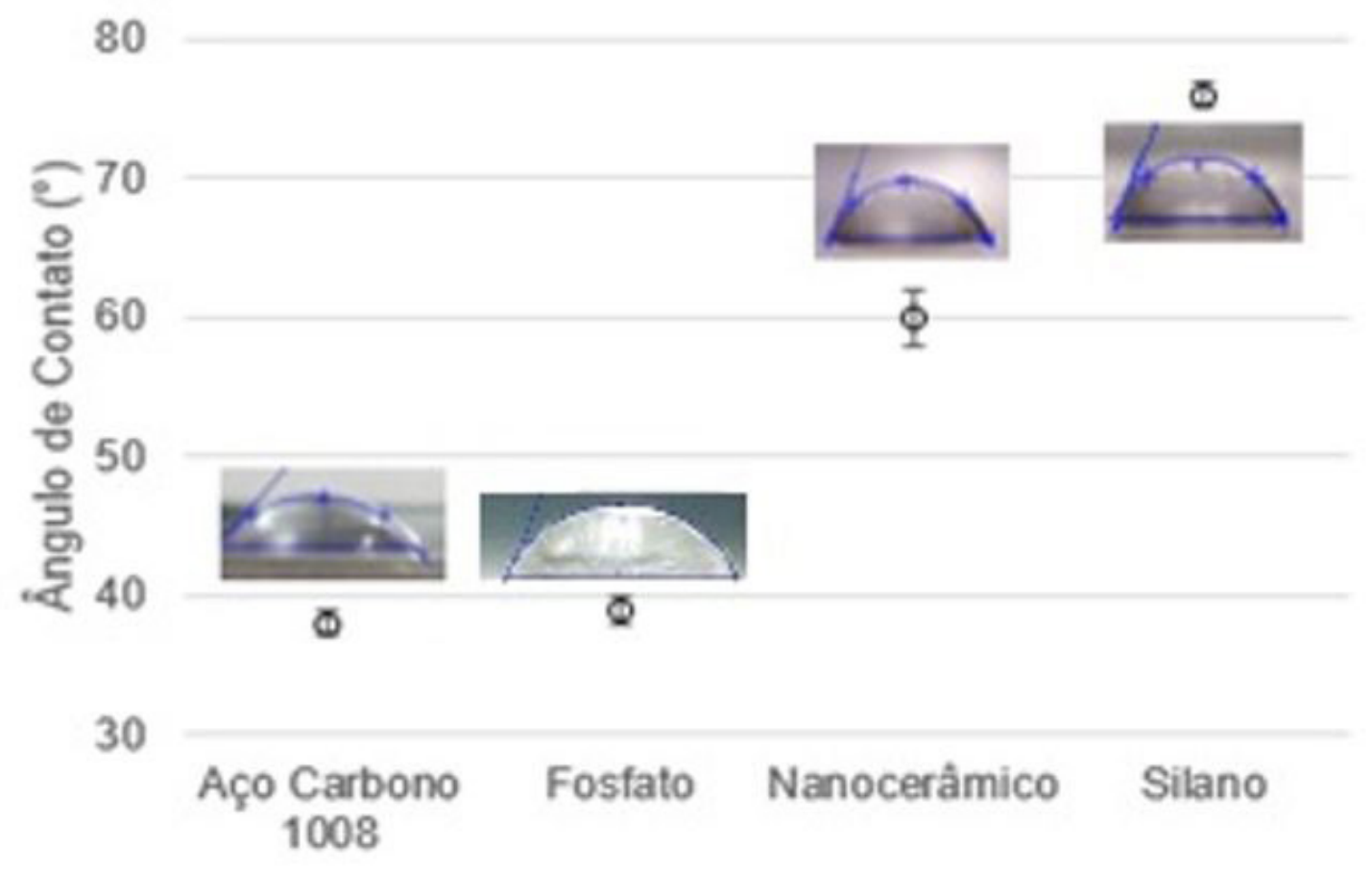

Figura 2. Resultados do teste de ângulo de contato para o aço AISI 1008 lixado e com aplicação de pré-tratamentos. 
grupos silanóis $(\mathrm{Si}-\mathrm{OH})$ indicando grupos hidrofílicos $[9,23]$. A formação de grupos $\mathrm{OH}$ é um parâmetro determinante na estrutura final da macromolécula e da funcionalidade do monômero, definida como o número de ligações que este pode estabelecer [9].

O filme de silano apresentou a menor banda para o grupo funcional $\mathrm{OH}$ (Figura 3c), em relação aos demais revestimentos, indicando que este pré-tratamento apresenta caráter mais hidrofóbico e reticulado em comparação aos sistemas fosfato e nanocerâmico, conforme os resultados encontrados na Figura 2. Isso permite concluir que a concentração de $\mathrm{OH}$ na estrutura do filme após a sua formação interfere na estabilidade e função barreira, pois sua presença promove caráter instável ao pré-tratamento. No estado de equilíbrio, a quantidade de grupos $\mathrm{OH}$ na superfície da partícula é um parâmetro importante, o qual limita o número de ligações covalente entre silano/metal indicando o grau de reticulação do filme [9].

Os resultados obtidos para o potencial de circuito aberto $(\mathrm{OCP})$ e polarização de todos os revestimentos estudados, seguem representadas na Figura $4 a$ e $4 b$.

$\mathrm{Na}$ Figura $4 \mathrm{a}$, observa-se uma rápida diminuição do valor do potencial para o aço lixado, indicando a ativação da superfície, a qual possuía a influência de óxidos protetores naturais. Quanto mais positivo forem os valores de potenciais, menos suscetível a corrosão é o material [24]. Por outro lado, a aplicação dos pré-tratamentos no aço implicou no deslocamento do potencial para valores mais positivos, em comparação ao aço lixado. Os três pré-tratamentos apresentaram comportamento semelhante nas primeiras 69 horas de imersão, seguido de uma queda no potencial até 189 horas, sendo mais acentuada para o filme de fosfato. Este efeito pode ser devido à descontinuidade do filme, Figura Ib, pemitindo que o eletrólito o permeie, entrando em contato com o substrato. Neste caso, a presença de íons fosfato como inibidores não tem efeito positivo sobre os valores de OCP [25]. No entanto os revestimentos nanocerâmico e silano, após 189 horas de imersão, apresentaram comportamento distinto. Isto indica que não é apenas a porosidade do filme que determina o comportamento eletroquímico do substrato, uma vez que ambos apresentaram valores de ângulo de contato próximos, mas também, a formação dos grupos característicos de cada filme, responsáveis pelo efeito barreira, conforme apresentado na Figura 3, ou seja, as reações anódicas e catódicas provenientes da interação entre substrato e pré-tratamento, influenciam de forma significativa o comportamento eletroquímico. Neste caso, a concentração maior do grupo $\mathrm{OH}$ (Figura $3 \mathrm{~b}$ ), proveniente do processo de redução do oxigênio para a formação do filme nanocerâmico, pode ter influenciado na variação mais negativa da curva de OCP em relação ao silano, uma vez que o grupo funcional $\mathrm{OH}$, por ser hidrofílico, prejudica o efeito barreira do filme, permitindo o contato do substrato com o meio $[26,27]$. Por outro lado, o filme a base de silano apresentou comportamento barreira, não apresentando variações significativas no valor de OCP, o que pode estar
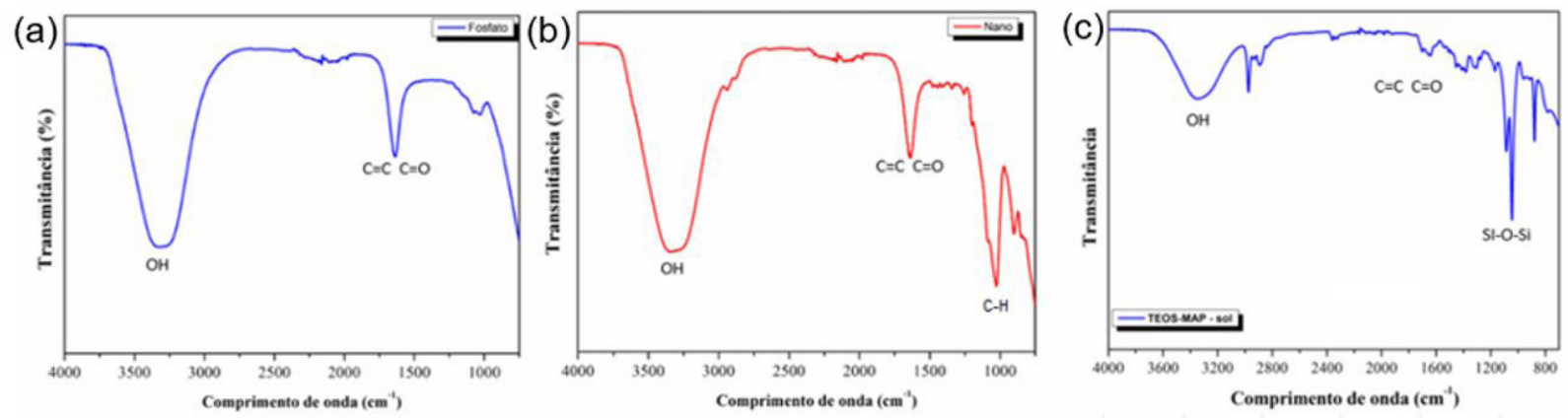

Figura 3. Representação dos picos encontrados no teste de FTIR para (a) solução de fosfato (b) solução de nanocerâmico e (c) solução de silano pós cura indicando a sua composição química.
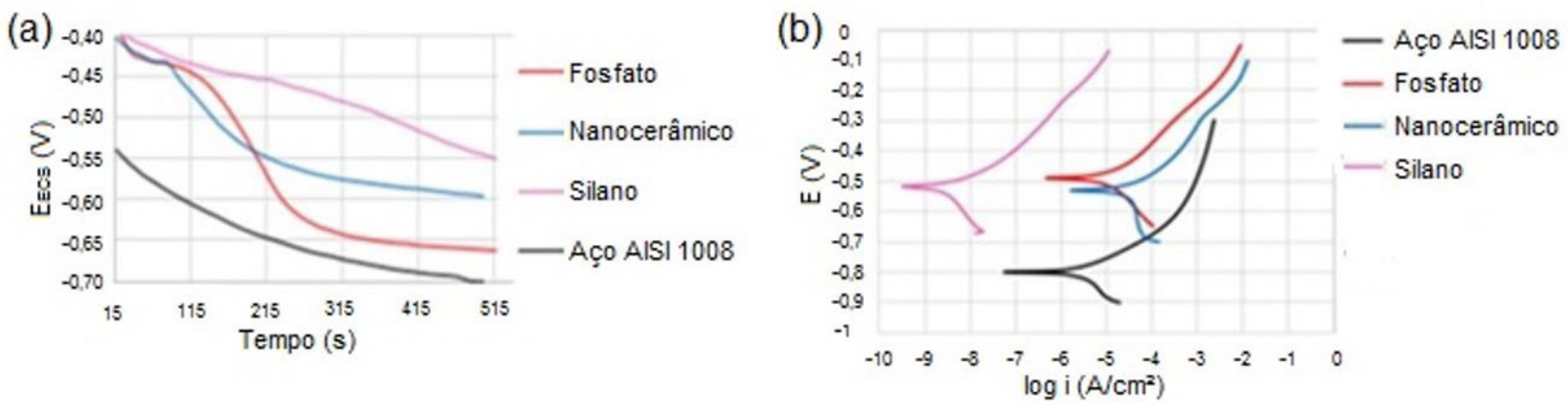

Figura 4. (a) Curvas do potencial de circuito aberto (OCP) e (b) Curvas de polarização para todos os pré-tratamentos aplicados sobre aço AISI 1008. 
relacionado, com a baixa concentração do grupo $\mathrm{OH}$, conforme discutido na Figura 3c, permitindo a formação de grupos $\mathrm{Si}-\mathrm{O}-\mathrm{Si}$, de caráter hidrofóbico e, por conseguinte, responsáveis pela propriedade barreira do filme.

Nas curvas de polarização (Figura 4b) foi possível observar que os três pré-tratamentos estudados proporcionaram um maior valor de potencial de corrosão em relação ao aço 1008 sem pré-tratamento. No entanto, o pré-tratamento de fosfato promoveu uma melhor resistência à polarização com uma menor corrente, em relação à amostra nanocerâmica, este comportamento pode estar relacionado com a presença de descontinuidades no filme nanocerâmico que contribuíram para a existência de caminhos preferencias para a permeabilidade do eletrólito e consequentemente influenciaram na resistência a corrosão [22]. Porém os sistemas nanocerâmico e fosfato, apresentaram valores de corrente maiores que o aço AISII 008, este comportamento pode estar relacionado com o sistema de conversão, o qual promove a aderência de tintas de proteção e decorativas pois modificam a rugosidade superficial do substrato, convertendo o mesmo em um óxido, hidróxido ou sal do metal, conforme relatado em outros estudos referentes a camadas de conversão $[28,29]$.

O filme de silano apresentou maior resistência à polarização e densidade de corrente de corrosão menor do que os demais pré-tratamentos. Isto pode estar associado ao fato dos filmes híbridos apresentarem caráter hidrofóbico, quando suficientemente reticulados (cross-linking), apresentando maior efeito de barreira, conforme discutido na Figura 2 e 3 , evidenciando a ação protetora desse pré-tratamento, conforme relatado por outros autores [30].

Os testes de Espesctroscopia de Impedância eletroquimica foram realizados com 24 e 72 horas de imersão em solução de $\mathrm{NaCl}$ 0,05 $\mathrm{M}$ e os resultados obtidos em função do gráfico de Bode estão representados na Figura 5.

Nas primeiras 24 horas, o pré-tratamento de fosfato apresentou dois fenômenos em média frequência devido a permeabilidade do eletrólito, e neste caso de forma significativa pois apresenta um ângulo menor, associado a produtos de corrosão citado nos ensaios eletroquímicos anteriores, e ao seu comportamento hidrofílico [3 I].

Os revestimentos restantes apresentaram apenas um fenômeno, o qual foi observado em média frequência para o aço e o pré-tratamento nanocerâmico (Figura 5a). O comportamento do nanocerâmico correlaciona-se com a permeabilidade do eletrólito através do filme, pelas irregularidades observadas no MEV (Figura Ib e Ic). O mesmo ocorre para $\circ$ aço, porém, neste caso, a permeabilidade do eletrólito acontece através do óxido de ferro. Como o ferro apresenta comportamento ativo, a permeabilidade
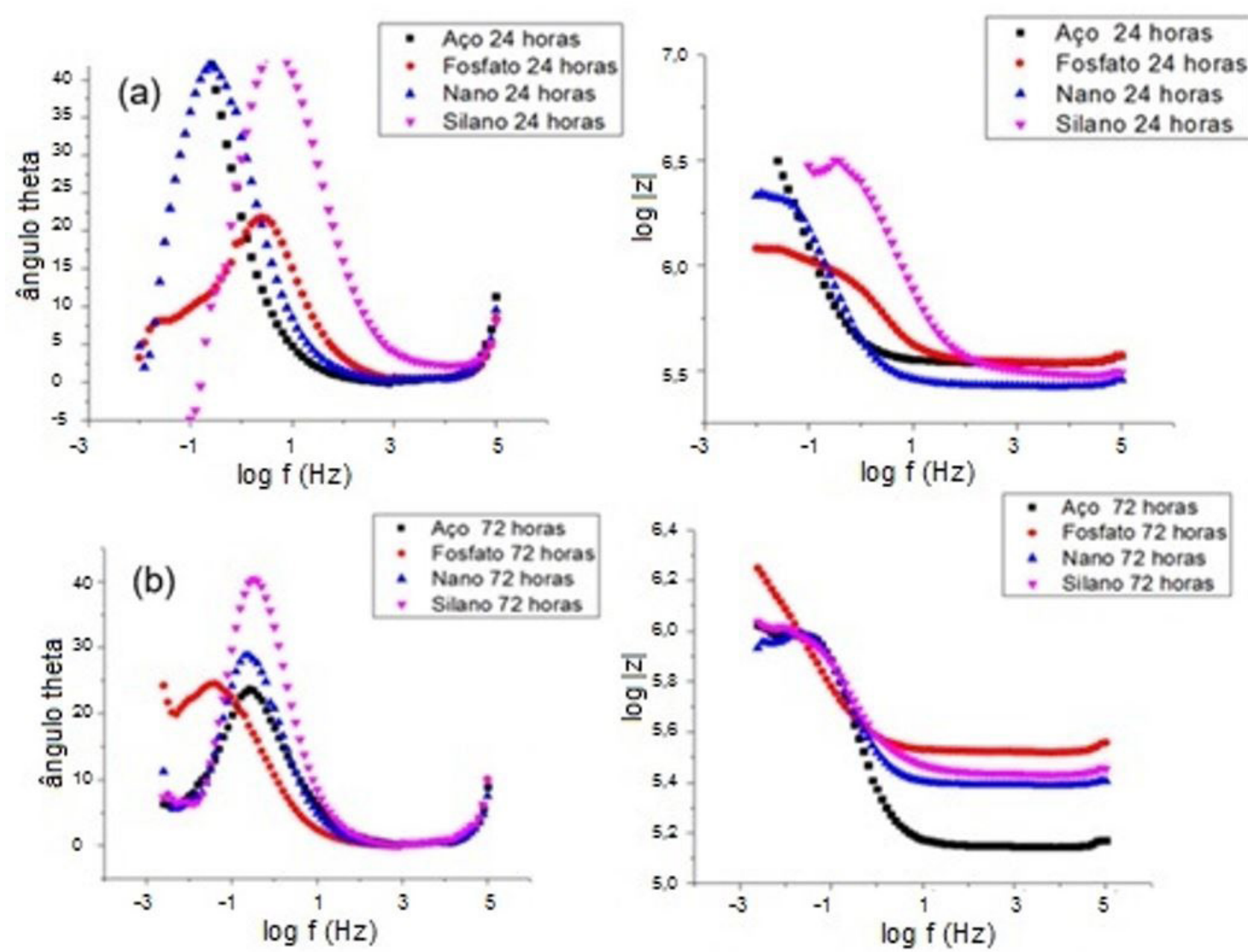

Figura 5. Diagrama de Bode do aço carbono 1008 e dos três pré-tratamentos estudados em (a) 24 horas e (b) 72 horas. 

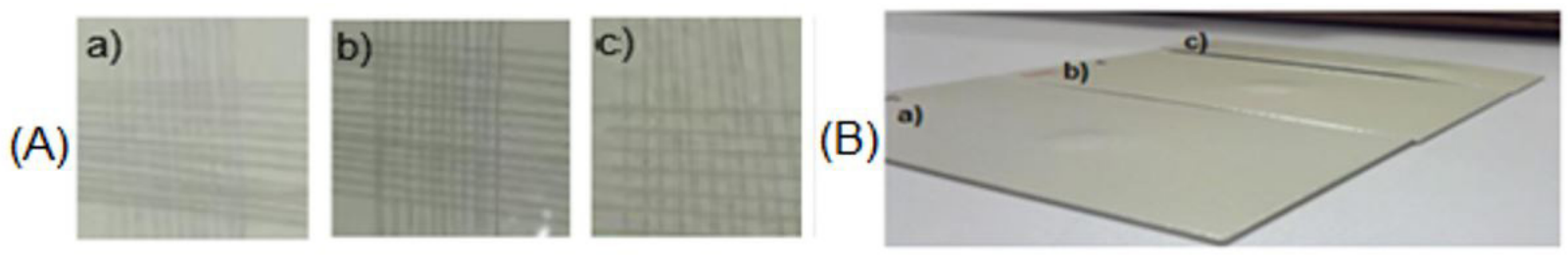

Figura 6. (A) Resultado do teste de aderência e (B) resultado do teste de impacto para os sistemas a) fosfato + tinta poliéster b) nanocerâmico + tinta poliéster e c) silano + tinta poliéster.
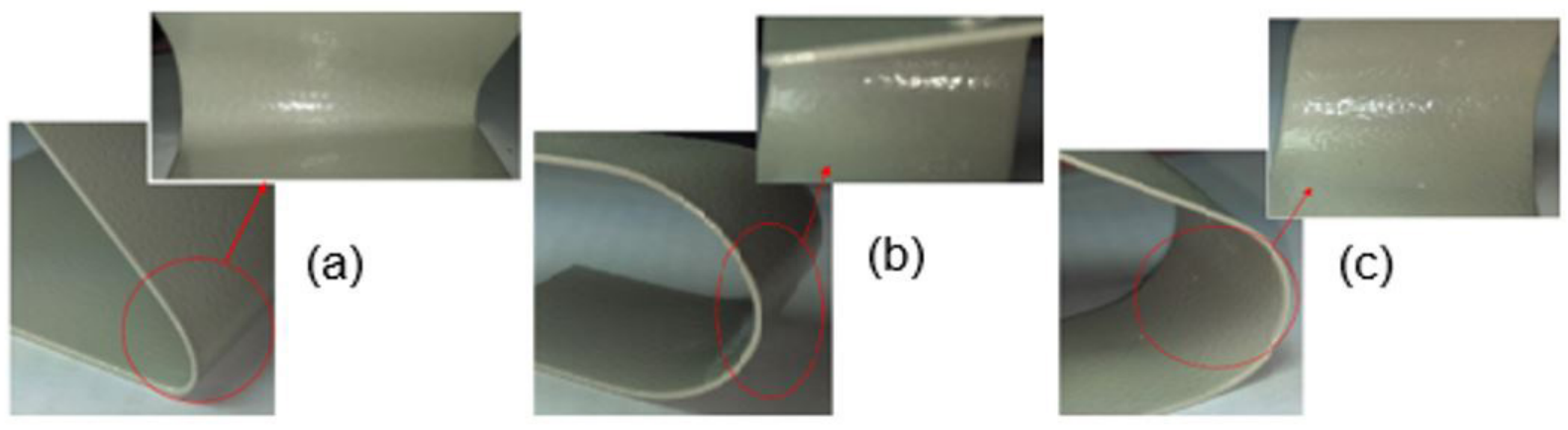

Figura 7. Representação visual da flexibilidade das amostras submetidas ao teste com mandril cônico: (a) fosfato + tinta (b) nanocerâmico + tinta e (c) silano + tinta.

pode ser atribuída à instabilidade do óxido ou formação de carbonato [32]. No caso do silano observa-se um fenômeno em alta frequência (Figura 5a), o qual está associado ao filme barreira decorrente da formação dos grupos $\mathrm{Si}-\mathrm{O}-\mathrm{Si}$, conforme discutido na Figura 3.

Em 72 horas de imersão, o fosfato apresentou comportamento semelhante ao aço 1008 , devido à permeabilidade do eletrólito através do filme de fosfato e de óxido de ferro. $O$ pré-tratamento nanocerâmico apresentou um decréscimo do valor de $|Z|$ e do ângulo de fase na região de média frequência (Figura $5 b$ ). Isto indica a penetração de espécies corrosivas através do filme, com o aumento do tempo, conferindo a este revestimento um menor efeito barreira [17]. Após 72 horas de imersão, o filme de silano manteve o mesmo comportamento, denotando o efeito protetor deste.

\subsection{Caracterização Mecânica}

A Figura 6A mostra 0 aspecto dos sistemas (pré-tratamento + tinta) após o teste de adesão. Não houve fissuras ou desplacamentos da tinta nas três amostras, o que significa que os parâmetros utilizados para a aplicação dos pré-tratamentos promoveram uma superfície adequada para o revestimento orgânico de tinta em pó poliéster.

A resistência ao impacto está fortemente relacionada ao grau de reticulação da tinta, ou seja, quanto melhor for a reação entre resina e o reticulante maior será a resistência do filme ao impacto. A radiação existente na própria estufa interage com as ligações covalentes ao longo do polímero e causa o rompimento das cadeias, o que facilita a deterioração das mesmas pela ação do oxigênio. $O$ antioxidante ao atuar contra esse processo de oxidação, evita que essas cadeias sejam quebradas e faz com que a rede polimérica formada pela reação entre resina e reticulante seja mais resistente tornando a tinta mais resistente ao impacto [33]. Os resultados do teste podem ser vistos na Figura 6B, em que é possível visualizar a inexistência de trincas ou rompimento da chapa nos três sistemas estudados. Isso garante a boa aderência de tinta nos três pré-tratamentos e um satisfatório processo de cura do filme.

Para uma tinta ser aprovada por resistência ao impacto deve apresentar resultados superiores a $50 \mathrm{~kg} / \mathrm{cm}$ (ASTM D 2794-93, 1999).

Para a análise de flexibilidade também deve-se levar em consideração as características da tinta utilizada. $O$ aspecto visual das amostras após serem submetidas a testes de flexibilidade por meio de um mandril cônico está representado na Figura 7 para os três sistemas estudados. Em análise visual macroscópica não foi observado a presença de trincas, fissuras e/ou desplacamento de tinta, corroborando com o ensaio de aderência, onde os três sistemas apresentaram bom desempenho.

Os resultados qualitativos se mostraram satisfatórios, uma vez que não comprometeram o revestimento. Desta forma, os três sistemas estudados apresentam boa resistência à flexibilidade em relação aos aspectos visuais. Para obter parâmetros significativos deve-se submeter as amostras a testes com microscópio óptico e verificar a interferência na estrutura dos pré-tratamentos de fosfatização, nanocerâmico e silano. 


\section{CONCLUSÃO}

O presente estudo mostrou que é possível optar por processos sustentáveis sem comprometer a qualidade do produto. Os resultados mostraram que os pré-tratamentos à base de silanos e nanocerâmicos, com posterior aplicação de tinta poliéster, obtiveram resultados semelhantes a fosfatização nos ensaios mecânicos, sendo que o silano apresentou desempenho superior nos ensaios físico-químicos e eletroquímicos em relação aos demais sistemas.

De uma forma geral, o pré-tratamento silano apresentou maior hidrofobicidade, com menores intensidades nas bandas $\mathrm{OH}$ nos testes de FTIR e um bom comportamento eletroquímico e mecânico, quando comparado aos demais pré-tratamentos testados.

Neste contexto, pode-se concluir que a proteção anticorrosiva é muito importante no âmbito econômico e ambiental, afim de diminuir o impacto ao longo dos anos e substituir processos nocivos ao meio ambiente, como a fosfatização. Pelo levantamento bibliográfico e pesquisa referente ao assunto apresentado, pode-se afirmar que o sistema silano apresentou maior resistência à corrosão e bom desempenho mecânico além, de ser um revestimento ambientalmente correto.

\section{REFERÊNCIAS}

I Curioni M. The behaviour of magnesium during free corrosion and potentiodynamic polarization investigated by real-time hydrogen measurement and optical imaging. Electrochimica Acta. 20 I4; 120:284-292.

2 Zheng L, Landon J, Matin NS, Liu K. Corrosion mitigation via a pH stabilization method in monoethanolamine-based solutions for post-combustion CO2 capture. Corrosion Science. 2016;106: I-I0.

3 Nijhuis CA, Reus WF, Whitesides GM. Molecular rectification in Metal-SAM-Metal Oxide-Metal junctions. Journal of the American Chemical Society. 2009; $131: 78$ I4-7827.

4 Qian M, Soutar AM, Tan XH, Zeng XT, Wijesinghe SL. Two-part epoxy-siloxane hybrid corrosion protection coatings for carbon steel. Thin Solid Films. 2009;517:5237-5242.

5 Kunst SR, Ludwig GA, Cardoso HRP, Santana JA, Sarmento VHV, Malfatti CF. Hybrid films with (trimethoxysilylpropyl) methacrylate (TMSM), poly (methyl methacrylate) PMMA and tetraethoxysilane (TEOS) applied on tinplate. Materials Research. 2014;17:75-8I.

6 Zubielewicz M, Kami ska-Tarnawska E, Kozłowska A. Protective properties of organic phosphate-pigmented coatings on phosphated steel substrates. Progress in Organic Coatings. 2005;53:276-285.

7 Mohseni M, Bastani S, Jannesari A. Influence of silane structure on curing behavior and surface properties of sol-gel based UV-curable organic-inorganic hybrid coatings. Progress in Organic Coatings. 2014;77:1191-1 199.

8 Phanasgaonkar A, Raja V. Influence of curing temperature, silica nanoparticles- and cerium on surface morphology and corrosion behaviour of hybrid silane coatings on mild steel. Surface and Coatings Technology. 2009;203:22602271.

9 Kunst SR, Cardoso HRP, Beltrami LVR., Oliveira CT, Menezes TL, Ferreira JZ, Malfatti CF. New sol-gel formulations to increase the barrier effect of a protective coating against the corrosion and wear of galvanized steel. Materials Research. 2015; I8:138-150.

10 Deflorian F, Rossi S, Fedrizzi L. Silane pre-treatments on copper and aluminium. Electrochimica Acta. 2006;5 I:60976103.

II Zhai Y, Zhao Z, Frankel GS, Zimmerman J, Bryden T, Fristad W. Surface pretreatment based on dilute hexafluorozirconic acid. In: Department of Defense. Proceedings of the Corrosion Conference; 2007; United States: Research Gate; 2007. p. I-I6.

12 Basiruddin SK, Saha A, Pradhan N, Jana NR. Advances in coating chemistry in deriving soluble functional nanoparticle. The Journal of Physical Chemistry C. 2010; I 14:I 1009-I I0I7.

13 Kunst SR, Cardoso HRP, Oliveira CT, Santana JA, Sarmento VHV, Muller IL, Malfatti CF. Corrosion resistance of siloxane-poly(methyl methacrylate) hybrid films modified with acetic acid on tin plate substrates: influence of tetraethoxysilane addition. Applied Surface Science. 2014;298: I- II.

14 Álvarez D, Collazo A, Nóvoa XR, Pérez C. The anticorrosive properties of sol-gel films doped with hydrotalcite nanoparticles applied on tinplate. Electrochimica Acta. 2014;131:137-I47.

I5 Popic JP, Jegdic BV, Bajat JB, Veljovic D, Stevanovic SI, Miskovic-Stankovic VB. The effect of deposition temperature on the surface coverage and morphology of iron-phosphate coatings on low carbono steel. Applied Surface Science. 201 I;257:10855-10862. 
16 Sheng M, Wang Y, Zhong Q, Wu H, Zhou Q, Lin H. The effects of nano-SiO2 additive on the zinc phosphating of carbon steel. Surface and Coatings Technology. 20I I;205:3455-3460.

17 Ramanatham E, Balasubramanian S. Synthesis and characterization of hexafluorozirconic acid powder and its application in nanoceramic coating. Surface and Coatings Technology. 2016;304:228-236.

18 Mohammadlooa HE, Sarabi AA, Alvani AAS, Sameiea H, Salimia R. Nano-ceramic hexafluorozirconic acid based conversion thin film: surface characterization and electrochemical study. Surface and Coatings Technology. 2012;206:4132-4I39.

19 Kunst SR, Ludwig GA, Santana JA, Sarmento VHV, Bertoli PP, Menezes TL, Ferreira JZ, Malfatti CF. Elaboração e caracterização de filmes híbridos siloxano-PMMA preparados pelo processo sol-gel sobre folhas de flandres: influência do pH do sol. Ciência e Tecnologia dos Materiais. 20I4;26:33-38.

20 Ding X, Xue L, Wang X, Ding K, Cui S, Sun Y, et al. Influence of bath PH value on microstructure and corrosion resistance of phosphate chemical conversion coating on sintered $\mathrm{Nd}-\mathrm{Fe}-\mathrm{B}$ permanent magnets. Journal of Magnetism and Magnetic Materials. 2016;416:247-255.

2I Shirtcliffe NJ, Mchale G, Atherton S, Newton MI. An introduction to superhydrophobicity. Advances in Colloid and Interface Science. 2010;161:124-138.

22 van Ooij WJ. Zhu D, Stacy M, Seth A, Mugada T, Gandhi J, Puomi P. Corrosion protection properties of organofunctional silanes. Science and Technology. 2005;6:639-664.

23 Amini R, Vakili H, Ramezanzadeh B. Studying the effects of poly (vinyl) alcohol on the morphology and anticorrosion performance of phosphate coating applied on steel surface. Journal of the Taiwan Institute of Chemical Engineers. 2016;58:542-551.

24 Stefanoni M, Angst U, Elsener B. Local electrochemistry of reinforcement steel -Distribution of open circuit and pitting potentials on steels with different surface condition. Corrosion Science. 2015;98:610-618.

25 Shi JJ, Sun W. Effects of phosphate on the chloride-induced corrosion behavior of reinforcing steel in mortars. Cement and Concrete Composites. 20I4;45:166- I75.

26 Andreatta F, Turco A, Graeve ID, Terryn H, Wit JH, Fedrizzi L. SKPFM and SEM study of the deposition mechanism of ZrTi based pre treatment on AA606I aluminum alloy. Surface and Coatings Technology. 2007;20 I:7668-7685.

27 Lunder O, Simensen C, Yu Y, Nisancioglu K. Formation and characterisation of Ti-Zr based conversion layers on AA6060 aluminium. Surface and Coatings Technology. 2004;184:278-290.

28 Donofrio J. Zinc phosphating. Metal Finishing. 2000;98:60-73.

29 Panossian Z, Santos CAL. Camadas fosfatizadas destinadas à conformação mecânica. Corrosão e Proteção. 20। 2;43(9):22-25.

30 Flis J, Kanoza M. Electrochemical and surface analytical study of vinyl-triethoxy silane films on iron after exposure to air. Electrochimica Acta. 2006;5 1:2338-2345.

31 Morks MF, Corrigan PA, Cole IS. Mn-Mg based zinc phosphate and vanadate for corrosion inhibition of steel pipelines transport of $\mathrm{CO}_{2}$ rich fluids. International Journal of Greenhouse Gas Control. 201 2;7:2 I8-224.

32 Veleva L, Alpuche-Aviles MA, Graves-Brook MK, Wipf DO. Comparative cyclic voltrametry and surface analysis of passive films grown on stainless steel 316 in concrete pore model solutions. Journal of Electroanalytical Chemistry. 2002;537:85-93.

33 Nedochetko MTS, Martins CR, Heyder S, Riella HG, Koslowski LAD. Estudo e avaliação de aditivos nas propriedades de tintas em pó do tipo poliéster em substratos metálicos e vítreos. Anais do $X X$ Congresso Brasileiro de Engenharia Química; 2014; Florianópolis, Brasil. São Paulo: Blucher; 20I4. p. I4|48-I4I55.

Submetido em: 6 Out. 2016

Aceito em: 31 Mar. 2017 